\title{
La formación docente por competencias en el contexto actual venezolano. Un acercamiento desde la voz de profesores universitarios
}

Teacher training by competences in the current Venezuelan context. An approach from the voice the college professors

Recibido: 26 de agosto de 2019 | Aprobado: 6 de diciembre de 2019

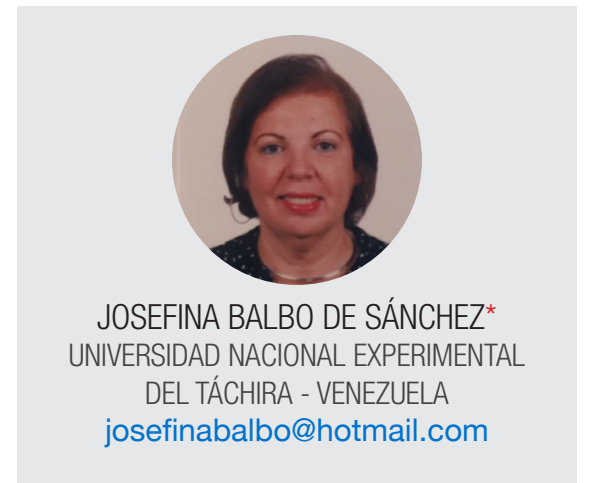

\section{Resumen}

En este artículo se muestran parte de los resultados de una investigación cuyo propósito fue explorar la perspectiva de profesores universitarios sobre la formación docente por competencias en Venezuela. E objetivo de este estudio consiste en reflexionar sobre los puntos de encuentro de las voces de los profesores universitarios acerca de la formación permanente en competencias en el contexto actual venezolano. Metodológicamente el estudio se apoyó en lainvestigación cualitativa, se enmarcó en el paradigma interpretativo, bajo la orientación de la fenomenología como método; en correspondencia con este, se seleccionó la entrevista en profundidad como técnica para la recolección de datos y los informantes fueron seis docentes de tres universidades públicas venezolanas. Los hallazgos señalan la necesidad de una formación docente basada en la enseñanza reflexiva y la integración de saberes y asumido como un proceso sistemático, continuo y permanente dirigido al desarrollo de competencias que permitan enfrentar la complejidad del acto educativo en el contexto venezolano, marcado por la crisis en aspectos económicos y sociales.

Palabras clave: formación docente; enfoque por competencias; educación universitaria; crisis económica y social

\section{Abstract}

This article shows part of the results of an investigation whose purpose was to explore the perspective of university professors on teacher training by competencies in Venezuela. The objective of this study is to reflect on the meeting points of the voices of university professors about on going training in skills in the current Venezuelan context. Methodologically, the study relied on qualitative research, was framed in the interpretive paradigm, under the guidance of phenomenology as a method; In correspondence with this, the in-depth interview was selected as a technique for data collection and the informants were six teachers from three Venezuelan public universities. The findings indicate the need for teacher training

\footnotetext{
Doctora en Pedagogía, Universidad de Los Andes - Venezuela. Docente e Investigadora, Categoría Titular, Dedicación Exclusiva, adscrita al Departamento de Estudios Sociales de la Universidad Nacional Experimental del Táchira - Venezuela. Coordinadora del Programa de Investigación Socio Humanístico (PISH-UNET). Para contactar a la autora: josefinaballbo@hotmail.com.
}

ISSN (en línea): 1814-4152 / Sitio web: http://cuaderno.pucmm.edu.do

CómO CITAR: Balbo, J. (2020). La formación docente por competencias en el contexto actual venezolano. Un acercamiento desde la voz de profesores universitarios. Cuaderno de Pedagogía Universitaria, Vol. 17, n. ${ }^{\circ}$ 33, enero-junio, pp.57-70 
based on reflective teaching and knowledge integration and assumed as a systematic, continuous and permanent process aimed at the development of skills that allow us to face the complexity of the educational act in the Venezuelan context, marked by the crisis in economic and social aspects.

Keywords: teacher training; competence approach; college education; economic and social crisis

\section{La formación del docente universitario y el enfoque por competencias}

La importancia de la formación de los docentes universitarios en competencias pedagógicas adquiere gran significado y se configura como un reto en esta sociedad del conocimiento cambiante, compleja y altamente tecnológica; para esto, se requiere que quienes gerencian los recintos universitarios asuman la responsabilidad de formar a sus profesores y estos, a su vez, decidan asumir la formación, ya que la educación superior conlleva, a juicio de Marcelo (1994), una:

Función social de transmisión de saberes, de saber hacer o del saber ser que se ejerce en beneficio del sistema socioeconómico o desarrollo y estructuración de la persona que se lleva a cabo bajo el doble efecto de una maduración interna y de posibilidades de aprendizaje, de experiencias de los sujetos, lo cual involucra a la estructura organizativa, que planifica y desarrolla las actividades de formación (p.175).

En esa misma línea, Hilarraza (2012) afirma que la formación de docentes universitarios debe fortalecer el desarrollo del pensamiento científico, innovador, reflexivo y crítico, sobre la práctica educativa mediante la integración tanto de teoría, como de práctica profesional, que permita vincular los saberes, pues no basta el saber hacer y conocer, se requiere también que dominen el ser, convivir y pensar.

Es necesario hacer referencia al enfoque de formación basado en competencias en el mundo universitario generado en Europa, denominado Proceso de Bolonia, que, como indica Beneitone (2009), nace por una intensa reflexión sobre la educación superior en el ámbito europeo, en torno a la creación de un ambiente de trabajo para que los académicos llegaran a puntos de referencia, comprensión y confluencia sobre estructuras educativas, titulaciones comprendidas, comparadas y reconocidas. Su resultado es un documento contentivo de las competencias genéricas y específicas que deben formarse, en el que se detalló el estado actual de la educación en América Latina, redes de discusión, debate sobre la educación superior en la región y redes temáticas de universidades europeas y latinoamericanas.

Estas iniciativas se instalan en el año 2004 en América Latina y el Caribe a través del Proyecto Tuning América Latina, para comenzar un diálogo conducente al intercambio de información, colaboración, calidad, efectividad y transparencia entre las instituciones de educación superior en 18 países latinoamericanos, con la premisa de tender puentes para el reconocimiento de las titulaciones con otras regiones del planeta desde cuatro grandes líneas: competencias genéricas y específicas, enfoques de enseñanza, aprendizaje y evaluación, créditos académicos y calidad de los programas.

El término competencias es un concepto polisémico y ambiguo. En esta investigación se aborda desde una visión amplia y abierta para el desempeño docente como un acto complejo y de prescripción abierta, entendido, según Le Boterf (2000), como una noción en que los docentes abordan un trabajo complejo (la enseñanza), que amerita un conjunto de acciones interligadas que no se resuelven aplicando un algoritmo o protocolo, sino que demanda competencias, iniciativas, transferencia, innovación y gestión y así enfrentar en el aula situaciones complejas eventuales y emergentes.

También Tobón (2013), al referirse a las competencias en el escenario de la educación superior, señaló que se estaban abordando desde un marco de descontextualización paradigmática, pues el constructo se originó en el paradigma teórico de la lingüística y se está aplicando a otras áreas en forma asistemática y desconexa; por ello, plantea que para usarse en educación superior, el término debe asociarse con otras fuentes teóricas, por estar articuladas a un sistema conceptual, de 
ahí que las define desde la complejidad; es decir, aborda las competencias desde el pensamiento complejo como método de construcción del conocimiento, basado en las relaciones entre las partes y el todo desde la continua organización orden-desorden, lo que implica abandonar la pretensión de tener ideas, leyes y fórmulas para comprender y explicar la realidad (Morín, 1999).

A este punto, es importante hacer mención de una clasificación más extendida en el campo educativo: las competencias genéricas y específicas. Las primeras son fundamentales para alcanzar la realización personal, gestionar proyectos y actuar en cualquier profesión, se forman desde la familia; son la esencia de la educación básica, media, técnicolaboral y educación superior, y se caracterizan por su importancia en la gestión de su plan formativo, el logro de la realización personal y el aprendizaje continuo, así como en la resolución de conflictos interpersonales y sociales (Tobón, 2013).

A saber de esto, las competencias genéricas comprenden: la autogestión de la formación (planifica procesos de formación acordes con el contexto, gestiona los recursos que requiere y realiza la formación hasta alcanzar las metas); trabajo en equipo (comprende el proceso de planificación de actividades en equipo y contribuye a generar una visión compartida); gestión de la información y del conocimiento (interpreta la información para generar conocimiento y procesa la información a través de determinada metodología); investigación (planifica proyectos de investigación, ejecuta el proceso, selecciona el método adecuado al objeto de estudio y socializa los resultados). En el mismo orden de ideas, Gimeno (2007) presenta una sistematización de las competencias genéricas para la formación docente y las clasifica de la siguiente forma: 1. Destrezas de comunicación, 2. Conocimientos básicos, 3. Destrezas técnicas, 4. Destrezas interpersonales.

Por otro lado, las competencias específicas, según Tobón (2004), son las propias de una determinada profesión, con alto grado de especialización, así como procesos educativos específicos; por tanto, los profesionales de la educación deben poseer las siguientes: dominar la teoría y metodología curricular para orientar acciones educativas; conocer a grandes rasgos los saberes de las disciplinas del área de su especialidad; saber diseñar estrategias de enseñanza y aprendizaje según contextos; conocer y aplicar en el acto educativo las teorías que fundamentan las didácticas generales y específicas; planificar procesos de evaluación con base en criterios determinados; elaborar e implementar recursos didácticos pertinentes al contexto; procurar el desarrollo del pensamiento crítico de los estudiantes y reflexionar sobre su práctica, con la intención de mejorar su quehacer educativo a través de la investigación en educación.

Por tanto, estos elementos representan una diversidad de habilidades y desempeños no jerarquizados, sino complementarios, que derivan en la comunión de acciones que exigen a los docentes formarse en ambas competencias para interactuar en la complejidad subyacente de su profesión, pues tal como se apreció, las competencias genéricas enriquecen el desarrollo de su labor mediante el trabajo en equipo, el liderazgo, gestión de la información e investigación, desde el respeto, ética y honestidad. Y las específicas, apuntan hacia el ejercicio de sus funciones en contextos determinados, en este caso, integrando saberes propios de cada disciplina, junto con el estudio y reflexión de estrategias de enseñanza, teorías didácticas, formas de evaluar, elaboración y uso adecuado de materiales didácticos; lo que significa, según los autores anteriormente citados, reflexionar sobre la práctica.

Este artículo se propone reflexionar sobre la necesidad de la formación permanente en competencias evidenciadas en las entrevistas a seis docentes de distintas universidades a la luz de los supuestos teóricos ofrecidos. Para lograr este objetivo, el trabajo investigativo se propone estructurar códigos a partir de las opiniones suministradas por los informantes para interpretar la tendencia representativa en la necesidad docente manifiesta. Además, el estudio procura sistematizar las informaciones ofrecidas y codificarlas de acuerdo a las dimensiones del contexto y a la formación docente.

Los acápites siguientes detallarán la metodología implementada y la caracterización del contexto actual atravesado por la crisis política y socioeconómica de Venezuela. Luego, se presentan los resultados, en los cuales se sistematizan las informaciones 
recabadas en las entrevistas y clasificadas de acuerdo a las unidades de análisis (contexto y formación docente). Más adelante, ofrecemos una reflexión sobre el punto de encuentro de las voces de los informantes con el fin de resignificar el compromiso docente, el cual se debe sobreponer a toda crisis. Por último, presentamos las conclusiones con una motivación a asumir la docencia a partir de la reflexión y el compromiso para enfrentar la complejidad del acto educativo en el contexto venezolano.

\section{Metodología}

Esta investigación se enmarcó en el paradigma interpretativo, bajo la orientación de la fenomenología como método de estudio (Latorre, Rincón y Arnal, 2003). Según Sandín (2013), resulta un método que parte del análisis de las experiencias vividas por las personas acerca de un fenómeno y se fundamenta en la descripción de los aspectos esenciales del significado trascendente, desde la visión de los investigados. De manera que el presente estudio se apoyó ampliamente en la investigación cualitativa, por cuanto la naturaleza del objeto de estudio orientó la interpretación de los fenómenos estudiados.

\section{Contexto de la investigación}

Se asumió como escenario del estudio tres universidades públicas y autónomas ubicadas en el estado Táchira, República Bolivariana de Venezuela: la Universidad Nacional Experimental del
Táchira (UNET), la Universidad de Los Andes (ULA) Núcleo universitario Dr. Pedro Rincón Gutiérrez y la Universidad Pedagógica Experimental Libertador (UPEL).

Técnicas e Instrumentos para la Recolección de la Información

Se seleccionó la entrevista en profundidad (Martínez, 2012; Ruíz, 1999) como técnica para la recolección de la información. Se utilizó la entrevista estructurada, como una forma de aproximarse a las percepciones, saberes, creencias y experiencias de los informantes en torno al objeto de estudio, razón por la cual fue necesario utilizar como instrumento un guion de preguntas abiertas. Las entrevistas fueron registradas en una grabación de audio y fueron transcritas para su posterior análisis; pero, en función de la veracidad de la información allí contenida, antes de iniciar la codificación abierta, las entrevistas fueron enviadas a los informantes, quienes las leyeron y aprobaron y en algunos casos, agregaron información adicional, que no manifestaron en el desarrollo de las sesiones.

\section{Informantes de Investigación}

Los informantes de esta investigación conformaron un grupo de seis profesores (6) de la UNET, ULA y UPEL. Los atributos o características asumidas para seleccionar a los informantes fueron: participación voluntaria y trayectoria profesional. Seguidamente se presentan en la tabla 1 las características de estos informantes.

Tabla 1. Características de los informantes

\begin{tabular}{|c|c|c|c|}
\hline Informantes & Universidad & Experiencia & \multicolumn{1}{c|}{ Código } \\
\hline \multirow{2}{*}{1} & UNET & $\begin{array}{l}13 \text { años de servicio, Doctor en Ingeniería Su inclusión se debe a } \\
\text { su experiencia como gerente en la formación y coordinador de un } \\
\text { programa de investigación. }\end{array}$ \\
\hline 2 & UNET & $\begin{array}{l}\text { Docente jubilado desde 1983. Magister en Educación y Currículo. Su } \\
\text { inclusión se debe a su experiencia como gerente de una dependencia } \\
\text { encargada de la formación docente, además de ser docente de } \\
\text { carrera. }\end{array}$ \\
\hline
\end{tabular}




\begin{tabular}{|c|l|l|l|}
\hline \multirow{3}{*}{3} & UNET & $\begin{array}{l}12 \text { años de servicio, adscrita a la Coordinación de Investigación Socio } \\
\text { Económica; doctora en Ciencias Gerenciales. Su inclusión se debe a } \\
\text { que, además de su condición de ingeniero, ha desarrollado estudios } \\
\text { en áreas afines a la educación y pertenece a un grupo de investigación } \\
\text { en ciencias sociales. }\end{array}$ & IC3 \\
\hline \multirow{5}{*}{} & UNET & $\begin{array}{l}10 \text { años de servicio, adscrita a la Coordinación de Investigación } \\
\text { Agropecuaria; doctoranda en Ciencias Gerenciales. Su inclusión se } \\
\text { realizó a manera de contraste, pues su formación y experiencia es solo } \\
\text { en su área disciplinar. (Investigador) }\end{array}$ & IC4 \\
\hline 5 & ULA & $\begin{array}{l}\text { Docente jubilado desde 1985, adscrito al Centro de Investigaciones } \\
\text { Geodidácticas de Venezuela; doctor en Ciencias de la Educación. } \\
\text { Amplia experiencia académica e investigativa en educación, formador } \\
\text { de formadores. }\end{array}$ & IC5 \\
\hline 5 & UPEL & $\begin{array}{l}\text { Educativas "Georgina Calderón" (UPEL); doctora en Educación. Amplia } \\
\text { experiencia académica e investigativa en educación, formador de } \\
\text { formadores. }\end{array}$ & IC6 \\
\hline
\end{tabular}

Procedimiento para el análisis de la información

El análisis de la información se apoyó en el proceso de codificación y categorización relativo a la inducción analítica, propia del enfoque de la Teoría Fundamentada expuesta por Strauss y Corbin (2002), con la intención de detallar los datos y conceptualizar, en términos de sus propiedades, dimensiones y relaciones desde la mayor rigurosidad posible. Es así que la revisión de entrevistas permitió reducir los datos a códigos, que a su vez reflejaron las frases emitidas por los informantes. Los resultados que emergieron se ordenaron en tres niveles: dimensiones, subcategorías y categorías. Ante esto, el proceso mencionado hizo necesaria la utilización del programa de aplicación para análisis cualitativo denominado Atlas-ti, versión 7.5.

\section{Resultados}

En este apartado se muestra el análisis de la información contenida en las entrevistas aplicadas a los seis docentes, específicamente, para este artículo se analizaron los componentes de las subcategorías Contexto y Enfoque por competencias de la categoría Formación docente. En la tabla 2 se aprecia de manera gráfica la articulación entre los códigos, dimensiones y las dos subcategorías, como fundamento de la categoría resultante denominada Formación docente, en atención del procedimiento planteado por Strauss y Corbin (2002).

Las particularidades de la situación actual promueven aspectos desfavorables que en lo institucional pudieran influenciar la formación docente, así como inconformidades, producto de la crisis y complejidad que limitan en la institución la formación docente. De allí que, la confrontación entre la evidencia empírica y la revisión teórica permitió la construcción de la categoría Formación docente, la cual emerge como una actividad en la que sus actores conviven en unión con una diversidad de necesidades en un contexto caracterizado por la crisis en aspectos económicos y sociales, que no les permiten asumir la formación de manera plena para ofrecer una docencia de calidad. 
Tabla 2. Categoría emergente formación docente

\begin{tabular}{|c|c|c|c|}
\hline Códigos & Dimensiones & Subcategorías & Categoría \\
\hline $\begin{array}{c}\text {-Contexto complejo. } \\
\text {-Crisis económica social- } \\
\text { Sobrevivencia. } \\
\text {-Fuga de cerebros en el sector } \\
\text { estudiantil y profesoral. } \\
\text {-Obstáculos externos. } \\
\text {-Limitaciones para responder a } \\
\text { los cambios. } \\
\text {-La crisis como oportunidad para } \\
\text { el cambio. }\end{array}$ & Complejidad y crisis & Contexto & \multirow[t]{3}{*}{ Formación Docente } \\
\hline $\begin{array}{c}\text {-Competencias } \\
\text { específicas del docente. } \\
\text {-Competencias genéricas del } \\
\text { docente. }\end{array}$ & Tipos de competencias & \multirow[t]{2}{*}{ Enfoque por competencia } & \\
\hline $\begin{array}{l}\text {-Docente reflexivo. } \\
\text {-Integración de los saberes en el } \\
\text { desempeño docente. }\end{array}$ & Saber Docente & & \\
\hline
\end{tabular}

De la construcción inductiva realizada en torno a la información suministrada por los docentes, podemos apreciar los diferentes códigos y dimensiones agrupados en la subcategoría Contexto. La dimensión Complejidad y Crisis es producto de la agrupación de elementos vinculados con la realidad inmediata que sugieren los entrevistados, en la que resaltan los códigos: contexto complejo, crisis económica social- sobrevivencia, fuga de cerebros en el sector estudiantil y profesoral, obstáculos externos, limitaciones para responder a los cambios y la crisis como oportunidad para el cambio.

Es así que, por ejemplo, los entrevistados señalan:

Producto de la crisis actual, se observa que se ha perdido el interés, el amor, la vocación por la profesión y se está en la universidad por un beneficio económico que hoy ya no es atractivo, por eso vemos a nuestros docentes haciendo otras labores y descuidando sus actividades [4:8]

No es un secreto que, en los tiempos complejos que hoy vivimos, los docentes no quieren o no están motivados para investigar, se limitan a dar sus clases [4:5]
El país en general y nosotros los docentes universitarios estamos atravesando una coyuntura altamente compleja [2:1]

Ser docente universitario hoy es muy diferente a otras décadas, la actualidad, la coyuntura política, social y económica que hoy vivimos [3:46]

Hoy la realidad es otra, se observa fuga de talentos y los que deciden quedarse deben recibir herramientas para ser empleadores y no empleados, hoy día creo las cosas han cambiado y ya debemos comenzar a incentivar la investigación y extensión [2:10]

Desafortunadamente nuestra misión se ha ido desdibujando y veo con mucha tristeza y preocupación cómo los docentes jóvenes, los contratados e incluso los fijos instructores y asistentes se están yendo del país, ya es recurrente ver concursos desiertos cuando antes los profesores se disputaban un cargo y los que se quedan se preocupan más por cubrir sus necesidades básicas [3:7] 
Mas por la situación del país que estamos viviendo en estos momentos y que pues por supuesto nadie es ajeno a ello y nadie escapa de ello; yo no cuento con la presencia activa de todo el personal, pues ya se hizo rutina el tiempo para salir a comprar para echar gasolina, para ir al colegio de los niños, para hacer cola, situaciones con la que en este tiempo hemos aprendido a convivir y a aceptar [2:161]

Nos hace repensar nuestro rol, no desfallecer y continuar esforzándonos por ofrecerles a nuestros estudiantes una educación de calidad, por consiguiente, la universidad debe generar políticas que consoliden las competencias necesarias de estos docentes en formación [3:47]

A saber de esto, es posible inferir que los docentes entrevistados desarrollan su labor en una realidad altamente compleja, la cual merma su intención investigativa y de formación, y en algunos casos se limitan solo a dar clases, obviando la responsabilidad de afrontar los retos que se les presentan como profesores universitarios, pues la crisis económica y social los obliga a dedicarse a otras tareas que les permiten acceder a beneficios económicos adicionales, pero en detrimento, al mismo tiempo, de las diferentes tareas académicas que les competen.

Hacer mención a la crisis actual que viven los docentes universitarios en Venezuela es acotar que, en los últimos años, según Martínez (2017), la educación dejó de ser una prioridad. La crisis, en opinión de este autor, es de tipo económica y social, enmarcada en un reiterado recorte presupuestario, bajos sueldos y escasa asistencia social, lo que, entre otras cosas, afecta a los docentes universitarios, quienes tratan de sobrevivir, en menoscabo del desarrollo y formación profesional y la investigación científica y académica, pues la prioridad es la satisfacción de las necesidades básicas (Barreto, 2017).

Es así que existe en la actualidad un contexto complejo en la institución, referido a obstáculos externos que no propician una labor docente de calidad, sino que, por el contrario, están ocasionando descontento, búsqueda de otros empleos y ausentismo, complejidad que en algunos casos se pueden ver como oportunidades para el cambio. Estas realidades complejas plantean la exigencia de una transformación en las universidades, en quienes las gerencian y en los docentes; lo cual se logra cuando se cambian los modos de pensar, de conocer, de vivir, por ende de formarse profesionalmente, siendo urgente redefinir el rol de la universidad y el de docentes universitarios.

Por otro lado, Albornoz (2012), señala que en la actualidad, en Venezuela, sigue vigente la necesidad de transformar las universidades, sobre todo, por el contexto político que se vive, hacerlas más humanas; en el sentido de que los docentes, pese a las dificultades y obstáculos que encuentran en el transitar de su profesión, se aboquen a “... dirigir acciones para formar a un nuevo republicano, con sentido crítico, reflexivo, participativo, conciencia y compromiso social..." (p.74); las universidades deben asumir también una postura de cambio, traducida en la generación de políticas de formación y seguimiento de la labor docente con indicadores de calidad que orienten a repensar la actividad docente a la luz de las propias complejidades y particularidades vividas por cada uno de ellos, para que las situaciones complejas que enfrentan se transformen en oportunidades para los cambios.

Desde esta visión se estipula la relación entre la complejidad y lo institucional, dado el input amplio y variado de posibilidades para asumir retos en la universidad, como la generación de políticas que determinen estándares de calidad y de seguimiento a la labor docente, para así cumplir con el rol que le fue asignado. Las situaciones manifestadas evidencian el contexto como un espacio complejo, en el que los docentes sienten una crisis sostenida, marcada por obstáculos externos que incluso han ocasionado fuga del talento a otros espacios para mejoras económicas y sociales; sin embargo, la presencia de esos obstáculos pudieran convertirse, según los informantes, en fortalezas institucionales, lo que devela el reconocimiento de la importancia de políticas de seguimiento a su labor.

Según García Llamas (1999), esto vendría a representar la lógica interna que opera dentro del constructo, como campo de conocimiento en el que los docentes aprenden y desarrollan competencias profesionales en un entorno complejo, dinámico 
y cambiante; con necesidades y obstáculos externos que ameritan cambios, orientados a adquirir habilidades para reflexionar sobre su práctica. Desde esta óptica, la formación docente es un entramado de relaciones y particularidades que lo convierte en un proceso complejo. Según Tobón (2004), consiste en lineamientos para generar condiciones pedagógicas para facilitar la adquisición de competencias a partir de la articulación de saberes.

A pesar de la crisis reflejada por la opinión de los informantes, estos también expresaron la necesidad de enfrentarla, y una manera es rescatando el rol docente, su amor, vocación, compromiso por enseñar y, además, la motivación para adaptarse a los cambios tecnológicos, así como sociales, que caracterizan el presente siglo, esto pasa por entender que sus responsabilidades van más allá de facilitar una jornada de clase, sino que se trata de vincular la enseñanza con la investigación y la formación para acoplarse a las nuevas exigencias y responder a los cambios.

La Subcategoría Contexto se presenta como un constructo emergente que derivó en este apartado y permitió aproximar los diversos elementos que caracterizan el significado de los docentes sobre el entorno inmediato desde el cual se desarrolla el proceso de formación. De manera que esta subcategoría permite inferir que la formación docente tiene lugar en un contexto ampliamente influenciado por la crisis que en la actualidad enfrenta la universidad venezolana y los diversos actores que hacen vida profesional dentro de ella, lo que exige una redimensión de su misión institucional, en función de la atención de cambios necesarios para la generación de estándares de calidad, y así hacerla competitiva en comparación con otras, pero además, para responder a las demandas de la sociedad, lo cual representa una reflexión que implica un horizonte formativo más allá de la simple transmisión de conocimientos.

Resalta así desde los anteriores testimonios que los informantes de la UNET, UPEL y ULA visualizan una universidad que, a pesar del contexto actual, caracterizado por la crisis económica, política y social, requiere redimensionar su significado. En ese sentido, Cortázar (2012) refleja que se debe seguir trabajando para el desarrollo de las universidades y su adecuación a las necesidades del entorno al devenir que proyecta el crecimiento del acervo científico y tecnológico, que tiene como norte la formación del talento, para que no se siga promoviendo la "... repetición de conceptos, desfasados en el tiempo, desarticulados disciplinariamente, fragmentados cognoscitivamente, ajenos o extraños a las necesidades de las mayorías nacionales..." (p.64).

Por esto, Albornoz (2012) opina que los docentes deben asumir los cambios y prepararse para responder a ellos, lo cual ocurrirá cuando asuman el rol docente como un proceso que no se consuma en la reproducción memorística de fórmulas y conceptos que solo refuerzan la dependencia intelectual, y que se planteen como meta responder al entorno actual, donde existen parámetros de calidad fijados por la comunidad académica internacional, en lo que concierne a la docencia y la investigación.

\section{Subcategoría Enfoque por competencias}

De la construcción inductiva realizada en torno a la información suministrada por los docentes, podemos apreciar los diferentes códigos y dimensiones agrupados en esta subcategoría. Es así que en esta sección dio lugar a la conformación de las dimensiones Tipos de competencias y Saber docente, que hicieron posible el surgimiento de códigos vinculados con las habilidades y desempeños que deberían caracterizar el conocimiento del docente como parte de su formación. La dimensión Tipos de competencias permitió establecer algunos elementos agrupados en códigos como Competencias genéricas del docente y Competencias especificas del docente, los cuales dan cuenta de funciones y habilidades de los profesionales que ejercen la docencia:

No menos importante, la competencia de lectura y escritura que les va a permitir consolidar las funciones como docentes e investigadores... [4:31]

Para realizar el acto didáctico debemos poseer una serie de competencias vinculadas con la acción docente, allí se encuentran seleccionar contenidos disciplinares, evaluar los resultados del aprendizaje, contribuir a la mejora de la docencia... [4:31] 
A pesar de que la universidad forme excelentes profesionales preparados en cada área disciplinar como es mi caso que egresé de una carrera de pregrado de la UNET, como es natural, no se me dieron todos los conocimientos que me permitieron luego ser docente... [2:6]

Es por ello que tengo conciencia que es un término [las competencias] con amplias acepciones, específicamente en el mundo educativo universitario, yo las defino como una integración de saberes: el saber que consiste en contenidos acumulados; el saber hacer que consiste en habilidades para realizar las tareas; y el saber ser, que se corresponde con el desarrollo personal, autoestima, manejo de conflictos, en fin, lo vinculado con la forma de comportarse del ser humano [2:115]

Todos esos esfuerzos en pro de cambiar ese paradigma que hemos tenido históricamente de preparar estudiantes para que queden en buenas empresas, por eso debemos prepararlos para que asuman nuevos retos, donde las cualidades personales son tan significativas como un buen record académico [2:8]

En términos de docente universitario, podemos hablar de competencias profesionales requeridas para un mejor desempeño, el cual está referido a las funciones que le competen. Específicamente hago referencia a la docencia y a la investigación; en este sentido, debe tener sólidos conocimientos en la disciplina que imparte (saber hacer); ser respetuosos con los alumnos, comprometido con los roles que asume, respetar sus ideas, ser flexible (saber ser); saber planificar sus clases, avaluar utilizando diferentes técnicas (saber conocer).

Tal como se puede evidenciar, los informantes atribuyen especial significado a las competencias que deben poseer los docentes universitarios, las cuales resultan en una serie de habilidades que les permiten, independientemente de la disciplina, mejorar su actuación en el rol que les compete. Tal como sugiere Pimienta (2012), las competencias permiten “...mejorar el desempeño o la actuación integral del sujeto, lo que implica conocimientos factuales o declarativos, habilidades, destrezas, actitudes y valores, dentro de un contexto ético..." (p. 2); es decir, integrar el saber conocer, el hacer y el ser en función de la solución problemas y situaciones que se presentan en su profesión.

De igual forma, Le Boterf (2000) expresa que un sujeto se hace competente cuando es capaz de saber actuar de forma pertinente en un contexto determinado, lo que implica elegir y movilizar recursos vinculados con el saber hacer (especificaciones técnicas que facilitan el desempeño efectivo en el puesto de trabajo) y saber actuar (recursos personales para resolver problemas del contexto). En las opiniones antes vistas de los informantes se muestra el interés por afianzar las competencias genéricas, es decir, aquellas habilidades del profesor de orden cognitivo y de orden motivacional que se manifiestan a través de las instrumentales, de orden metodológico o de procedimiento, tales como la capacidad de análisis y síntesis, de organización y planificación, y de gestión de información; las personales, como capacidad para el trabajo en equipo, la habilidad para el manejo de las relaciones interpersonales, el compromiso ético y las sistémicas, que se manifiestan en el aprendizaje autónomo, la adaptación a nuevas situaciones, la creatividad y el liderazgo; que, en opinión de Tobón (2004), son necesarias para mejorar el desempeño de los docentes universitarios.

En el mismo orden de ideas, Zabalza (2007) enfatiza las competencias profesionales específicas que deben mostrar en el desempeño los docentes universitarios, y destaca especialmente la competencia comunicativa, pues según este autor "...si hubiera que condensar en pocas palabras la imagen de un buen profesor, tendríamos que recoger esa idea, que sabe explicar bien su materia..." (p.82); lo cual parece confirmarse desde las expresiones de los informantes. Ellos, además, resaltan la importancia de manejar la lectura y la escritura, competencia comunicativa que se desarrolla en la medida en que se mantiene contacto con los textos y su producción y se afina en la medida que nos hacemos expertos en el discurso disciplinar, mediante la investigación, por ejemplo.

Por su parte, la dimensión Saber docente reúne las diferentes evidencias apreciadas en cuanto al conocimiento de los profesores, agrupadas en este caso en los códigos 1) Docente reflexivo: 
Los que nos quedamos tenemos la responsabilidad de mejorar nuestra práctica de aula, tomar en consideración las limitaciones de los estudiantes, sus estilos de aprendizaje, conocimientos previos, formarnos en posturas de enseñanzas basadas en el constructivismo como forma de generar conocimientos, donde el docente motive y fomente el aprendizaje significativo de los alumnos [1:59]

Se requiere una reflexión y toma de consciencia para que los que somos ingenieros reconozcamos la importancia y la complejidad de la práctica docente y se nos estimule a participar en programas de formación y actualización que respondan a necesidades personales y propias de la UNET en los ámbitos pedagógico, profesional, personal y social [2:14]

Ser un docente reflexivo pasa por el hecho de entender que estamos en una época, en primer lugar, de una explosión del conocimiento; en segundo lugar, de un avance extraordinario de la ciencia y la tecnología y, en tercer lugar, de una revolución comunicacional... [3:55]

Y 2) Integración de los saberes en el desempeño docente:

Se requieren habilidades para saber hacer, actitudes y valores que se corresponden con el ser, es enfilar los saberes en el aula de clase para realizar en forma idónea su función [1:37]

Se les debe enseñar y mostrar los beneficios de realizar procesos de formación y debe tocar lo pedagógico, lo investigativo, lo personal y lo tecnológico [4:13]

Es posible interpretar la tendencia representativa asociada con el saber docente que, desde la información obtenida en las entrevistas, se vincula con la enseñanza reflexiva, entendida como aquella que requiere una toma de conciencia, particularmente en los que no son docentes de profesión, sobre la importancia y la complejidad de la práctica del profesor, lo cual implica que en el contexto actual caracterizado por la explosión del conocimiento, avance de la ciencia, tecnología y la revolución comunicacional, amerita que los docentes universitarios mejoren su práctica de aula desde la consideración de las limitaciones de los estudiantes, sus estilos de aprendizaje, conocimientos previos, con la intención de motivar y fomentar el aprendizaje significativo, a pesar de una situación económica y social de crisis.

En ese orden de ideas, Boggino y Rosekrans (2004) expresan que los profesores deben formarse para convertirse en personas altamente reflexivas, capaces de transformar la enseñanza desde los conocimientos, creencias, valores e ideologías de los alumnos, pues la globalización y vertiginosidad del conocimiento, no permite "... seguir enfrascados en las propias perspectivas, sino que trata de comprender a los otros, a conocer lo diferente de las propias percepciones, a autoevaluar los conocimientos..." (p.141); por cuanto la docencia representa una de las profesiones que más exige el desarrollo del pensamiento complejo y la reflexión, desde la reformulación de sus propios esquemas cognitivos.

De igual forma, se interpreta la tendencia representativa asociada con el saber docente, que desde la información obtenida en las entrevistas, se vincula con la integración de saberes; lo que se traduce en la necesidad de desarrollar habilidades para el saber hacer, además de actitudes y valores que se corresponden con el ser, con la intención de enriquecer los saberes en el aula de clase y coadyuvar en la idoneidad en su desempeño; para lo cual deben formarse en lo que concierne a lo pedagógico, lo investigativo, lo personal y lo tecnológico.

Desde esta visión, se estipula que la dimensión Saber docente se perfila en este estudio, desde las percepciones de los informantes y la revisión teórica, como el dominio de los docentes sobre lo que enseñan, así como la habilidad de combinar lo teórico y lo práctico, que en opinión de Díaz (2006), se logra cuando conocen bien lo que enseñan y saben además cómo enseñarlo; pero a pesar ello, aún se observa que la actuación de los docentes se rige por un currículo orientado a los objetivos, en el cual "...la práctica supera a la discusión y reflexión teórica..." (p.20). Ante esto, se aprecia la necesidad de resignificar los saberes docentes, pues se confirma que los profesores tienen el compromiso de desarrollar competencias para la enseñanza que permitan enriquecer esos saberes desde la valoración permanente del acto mismo de enseñar. 
El constructo emergente subcategoría Enfoque por competencias refiere los hallazgos aproximados en las dos dimensiones anteriores, en las que se aprecian diversas tendencias asociadas tanto con el conocimiento de los profesores, como con las habilidades y desempeños como parte de su perfil para el ejercicio de la enseñanza. Así pues, los tipos de competencias están descritos desde las habilidades necesarias que deben poseer los docentes universitarios en su actuación cotidiana, que pasan por implicar sus conocimientos declarativos con valores, actitudes y destrezas, dentro de un contexto ético; es decir, integrar el saber conocer, el hacer y el ser, para resolver problemas que se presentan.

De los saberes docentes destaca la docencia reflexiva y la integración de los saberes en el desempeño docente como mecanismos para transformar la enseñanza, vinculando la teoría y la práctica desde la posibilidad de integración entre el saber ser, saber conocer y saber hacer, en la búsqueda de un ser humano que responda de manera pertinente a las profundas transformaciones del contexto actual.

La red de conceptualizaciones que dieron lugar a la categoría Formación docente refleja una tendencia significativa específicamente orientada hacia la conformación de un perfil basado en el enfoque por competencias, en respuesta a los múltiples desafíos que se les exigen a los docentes desde el contexto de crisis actual, lo cual guarda relación con los argumentos expuestos por Beneitone (2009), quien señala que el enfoque por competencias debe seguir asumiéndose por la universidad como una meta prioritaria para el fomento de la cristalización de los perfiles profesionales y académicos, con la intención de que los alumnos desarrollen más herramientas acordes con las necesidades de la sociedad y los posibles retos de empleos futuros.

Esto último también representa otro importante elemento apreciado en las evidencias, es decir, el docente debe poseer las competencias necesarias en cuanto la integración de saberes y el desarrollo de estados reflexivos con el fin de transformar la enseñanza; de esa forma, los hallazgos sugieren que los informantes poseen la convicción de continuar su formación en competencias, para a su vez, poder formar a sus alumnos bajo el enfoque por competencias, a pesar de la crisis actual que vive Venezuela, la cual dificulta el desarrollo de procesos de formación en la universidad.

\section{Reflexión acerca de los resultados}

Es importante destacar que el contexto está permeado, según los informantes, por la crisis económica social actual, que les exige adaptarse y enfrentar los cambios desde una serie de limitaciones para ello. Es perentorio, de esa forma, seguir desarrollando la formación por competencias, descrito por los informantes como actitudes, habilidades y destrezas que les permiten integrar los saberes para el mejor desempeño, razón por lo cual resulta necesario e importante un proceso de formación progresivo, sistemático y permanente dirigido a la consolidación de competencias que permitan enfrentar la complejidad del entorno social y político del país y la complejidad del acto educativo para mejorarlo.

Las circunstancias externas hacen obligatoria la revisión estructural de la universidad para adecuarla a la dinámica actual, caracterizada por la crisis y la transformación, lo cual se suma adicionalmente a la importancia de la revisión curricular, especialmente desde el enfoque por competencias, lo que exige, por ejemplo, la necesidad de revisar programas, cátedras, formas de abordar la enseñanza, evaluación, procesos de formación. Al contrastar las opiniones de los informantes con los supuestos teóricos considerados, se muestra cómo lo relatado coincide con Albornoz (2012), quien afirma que en Venezuela es una exigencia iniciar con las obligaciones sociales de la universidad, con la intención de precisar índices y niveles de productividad, pues según este autor, “....al menos en el área académica no estamos en la competencia..." (p.409).

Los informantes de la UNET reconocieron que en razón de su área formativa, el campo de la ingeniería no posee las competencias necesarias para mejorar la enseñanza, por lo cual resaltaron la necesidad de la dinámica formativa asociada con el desarrollo de habilidades, actitudes y desempeños, orientada principalmente al requerimiento de formación de competencias genéricas y específicas, que les permitan ejercer la docencia desde la integralidad y articulación del saber hacer, conocer, ser, 
convivir, para favorecer la formación de los futuros profesionales.

Para los profesores de la ULA y UPEL, el enfoque por competencias es el más indicado para asumir los procesos de formación de estudiantes y docentes en servicio; para el primer informante, la complejidad en el acto de enseñar exige que los docentes se formen en herramientas, destrezas y habilidades para mejorar la enseñanza, desde la reflexión. Para el informante de UPEL, el enfoque por competencias es asumido en el currículo de la universidad y se forma a estudiantes y docentes en servicio en competencias dirigidas a mejorar la enseñanza, con énfasis en las competencias tecnológicas, investigativas y de desarrollo personal, sobre todo, se enmarcan en lo ético, pues están conscientes de que no son entes aislados, sino que interactúan en un contexto complejo de crisis y de limitaciones externas que exige un despliegue de valores, compromiso y honestidad, vocación y amor, frente a la difícil tarea que decidieron asumir.

El punto de encuentro de los seis informantes de las universidades seleccionadas fue reconocer que en cualquier área disciplinar se debe asumir un proceso de formación permanente en competencias profesionales, que involucre el saber ser, hacer y conocer; es decir, asumir la enseñanza desde la integralidad de saberes, fortaleciendo la disciplina con la investigación, enmarcada en un ejercicio de respeto por el otro.

\section{Conclusiones}

Los docentes entrevistados señalan la necesidad de continuar la formación por competencias, tomando en cuenta el contexto complejo en el que se encuentra la situación económica y social venezolana y en particular, la educación. En este sentido, ven la crisis como una oportunidad para la transformación educativa en la universidad, desde la vocación y el compromiso como docentes. Desde esta representación, la formación por competencias emerge como una posibilidad formativa asociada al desarrollo de habilidades, actitudes y desempeños que asume la integración de los saberes, como vía para propender a la capacitación de docentes y alumnos integrales, bajo la premisa de que para enseñar y formar futuros profesionales, es necesario la articulación de los saberes fundamentales vistos en el saber, ser, hacer y conocer, con la intención de responder a las características complejas, dinámicas, así como cambiantes, de la sociedad del conocimiento actual en general, y en particular, la situación venezolana.

La formación desde el enfoque basado en competencias representa un proceso que puede enriquecer la acción reflexiva de los docentes como profesionales que ejercen la enseñanza capaces de reconocer sus debilidades en el ejercicio didáctico, que desean formarse en temas vinculados con el arte de enseñar, que están dispuestos a investigar sobre temas inherentes al acto educativo, pero, sobre todo, hacer de la enseñanza un entramado en el que converjan los diferentes saberes, para así potenciar el desarrollo de los principales actores del hecho educativo de manera integral, es decir, los estudiantes.

En este sentido, por ejemplo, Marcelo (1994) señala que los docentes deben ser formados desde sus etapas iniciales, sobre todo para que adquieran habilidades vinculadas con el arte de enseñar, aclarando que los encargados de impartir la formación deben estar formados en el conocer, hacer y ser; de esa manera, también serán formados los futuros egresados y esta concatenación de los saberes les permitirá actuar de manera competente frente a cualquier situación laboral que se les presente, lo cual es equivalente a formarlos en los ámbitos físico, mental, social y espiritual.

En definitiva, la formación por competencias se presenta como una dinámica formativa que posibilita el desarrollo de habilidades docentes desde la integralidad de saberes científicos, físicos, mentales, sociales y espirituales, y esto involucra de forma permanente el cuestionamiento y, por ende, la reflexión sobre el desarrollo de la enseñanza; lo que, sin duda, favorecería el desempeño de los docentes frente a los retos impuestos por el contexto particular en el que se encuentra la educación venezolana.

\section{Referencias bibliográficas}

Albornoz, O. (2012). Las múltiples funciones de la universidad: crear, transferir y compartir conocimiento. San Cristóbal: Fondo Editorial Simón Rodríguez. Lotería del Táchira. 
Ávalos, B. (2007). Formación docente continua y factores asociados a la política educativa en América Latina y el Caribe. BID.

Bacarat, M. y Graziano, N. (2002). ¿Sabemos de qué hablamos cuando usamos el término competencia? Bogotá: Sociedad Colombiana de Pedagogía.

Barreto, J. (2017, noviembre 5). La educación universitaria en Venezuela. Una crisis anunciada. [Entrevista a V. Márquez y B. Sharifker]. El Nacional, C-4.

Beneitone, P. (2009). El proyecto Tuning América Latina. Ponencia presentada en el Seminario Taller Centroamericano sobre desarrollo curricular basado en competencias y evaluación de calidad de la educación superior, Argentina.

Boggino, N. y Rosekrans, K. (2004). Investigaciónacción: reflexión crítica sobre la práctica educativa. Argentina: HomoSapiens.

Cortázar, J. (2012). La universidad venezolana a comienzos del siglo XXI. Conflictos, dilemas $y$ retos. La universidad debate. Venezuela: Fondo Editorial Simón Rodríguez.

Díaz, V. (2006). Construcción de saber pedagógico. San Cristóbal: Litoformas

García Llamas, J. (1999). Formación del profesorado. Necesidades y demandas. Madrid: La Muralla.

Gimeno, J. (2007). El curriculum: una reflexión sobre la práctica. Madrid: Editorial Morata.

Hilarraza, J. (2012). Investigación pedagógica. Un aporte a la gestión de la formación docente desde un punto de vista socio cultural. Revista Didáctica y Educación de Venezuela, 3(1), 1117.

Latorre, A., Rincón, D. y Arnal, J. (2003). Bases metodológicas de la investigación educativa. Barcelona: Experiencia.
Le Boterf, G. (2000). Ingeniería de las competencias. Barcelona: Gestión 2000.

Ley de Universidades. (1970). (1970, Septiembre 8). Gaceta Oficial de la República de Venezuela, 1429 (Extraordinario), septiembre 15, 1970.

Marcelo, C. (1994). Formación del profesorado para el cambio educativo. Barcelona: Promociones y Publicaciones Universitarias.

Martínez, P. (2017). Los problemas que devoran a las universidades venezolanas. [Entrevista a A. Belmont]. El Nacional, C-4.En: https://runrun. es/investigacion/295473/los-problemas-quedevoran-a-las-universidades-venezolanas/

Martínez, M. (2012). Nuevos fundamentos en la investigación científica. México: Trillas.

Morín, E. (1999). La cabeza bien puesta. Buenos Aires: Nueva Visión. Narcea.

Perrenoud, P. (1999). Diez nuevas competencias para enseñar. Barcelona: Grao.

Pimienta, J. (2012). Las competencias de la docencia universitaria. México: Pearson.

Ruíz, J. (1999). Metodología de la investigación cualitativa. España: Universidad de Deusto.

Sandín, M. (2013). Investigación cualitativa en educación. Fundamentos y tradiciones. Madrid: McGraw-Hill.

Schon, D. (1992). La formación de profesionales reflexivos. Barcelona: Paidós.

Strauss, A. y Corbin, J. (2002). Bases de la investigación cualitativa. Técnicas y procedimientos para desarrollar la teoría fundamentada. Medellín: Universidad de Antioquia.

Tobón, S. (2004). Formación basada en competencias. Pensamiento complejo, diseño curricular y didáctica. Bogotá, Colombia: Ediciones Ecoe.

Tobón, S. (2013). Formación integral y competencias. Colombia: Ecoe. 
Ugueto, M. y Cardozo, N. (2014). La gestión de recursos humanos por competencias. San Cristóbal: FEUNET.

Zabalza, M. (2007). Competencias docentes del profesorado universitario. Calidad y desarrollo profesional. Segunda edición. Madrid, España: Ediciones Narcea S.A. 